\title{
Histopathological features of gastrointestinal mucosal biopsies in children with juvenile idiopathic arthritis
}

\author{
Judith Pichler ${ }^{1,2}$, Christina Ong ${ }^{2,3}$, Neil Shah ${ }^{2,4}$, Neil Sebire ${ }^{5}$, Fevronia Kiparrissi ${ }^{2}$, Osvaldo Borrelli ${ }^{2}$, Clarissa Pilkington ${ }^{6}$ and \\ Mamoun Elawad ${ }^{2}$
}

BACKGROUND: The association between inflammatory bowel disease and joint involvement is well established. There is a paucity of data describing histopathological features of the gut in relation to juvenile idiopathic arthritis (JIA).

METHODS: We retrospectively identified 33 (21 male) children aged 3-16 y with JIA (11 with oligoarthritis, 5 with polyarthritis, 8 with systemic onset arthritis, 8 with enthesitis-related arthritis (ERA), and 1 with psoriatic arthritis) with significant gastrointestinal (Gl) symptoms who underwent upper and/or lower endoscopy. The histopathological findings were reviewed in addition to presence of autoantibodies and concomitant treatment.

RESULTS: The most common GI indications for endoscopy were persistent abdominal pain (14/33 (42\%)) and diarrhea $(10 / 33(30 \%))$. Of the 33 children, $28(85 \%)$ had gut mucosal inflammation, mostly affecting the colon (80\%). Active inflammation of the gut was found in 5 of 28 (17\%) children, and 15 of $28(53 \%)$ children showed mild nonspecific inflammation. Eight patients (27\%) had predominantly an eosinophilic infiltrate. Twenty-six patients had previously received treatment for JIA. There was a negative association with the use of immunomodulators and the presence of eosinophil inflammation.

CONCLUSION: The majority of children with JIA and GI symptoms have histological evidence of mild nonspecific inflammation, but some having active colitis and prominent eosinophil infiltrate.

J uvenile idiopathic arthritis (JIA) is the most common chronic rheumatic disease in childhood and is an important cause of short- and long-term disability. It is a clinical diagnosis made in a child younger than $16 \mathrm{y}$ of age with arthritis (defined as swelling or limitation of motion of the joint accompanied by heat, pain, or tenderness) for at least a duration of $6 \mathrm{wk}$, with other identifiable causes of arthritis excluded, the physical findings may be associated with raised inflammatory markers and positive findings on radiology. Studies in developed countries have reported a prevalence that varies between 16 and 150 per 100,000 children (1). The International League of Associations for Rheumatology classification of JIA includes seven subtypes: systemic onset JIA, oligoarticular JIA, polyarticular rheumatoid factor (RF)-positive JIA, polyarticular RF-negative JIA, enthesitis-related JIA, psoriatic JIA, and others or unclassified (2).

Arthritis is a common extraintestinal manifestation of several conditions including inflammatory bowel disease (IBD), bacterial or parasitic infections of the gut, coeliac disease, and pseudomembranous colitis. Arthritis is the most frequent extraintestinal manifestation, occurring in $7-25 \%$ of children with IBD that may precede the onset of gastrointestinal (GI) symptoms by years $(3,4)$. It appears to be more prevalent in patients with large bowel than those with small bowel disease and in those with complications such as abscesses, pseudomembranous polyposis, perianal disease, massive hemorrhage, erythema nodosum, stomatitis, uveitis, and pyoderma gangrenosum (5).

Crohn's disease (CD) and ulcerative colitis, collectively known as IBD, are chronic idiopathic conditions of the GI tract with nearly one quarter of cases having onset during childhood or adolescent years (6). Several studies in adult populations have described increased comorbidity with other immune-mediated diseases including asthma, arthritis, rheumatoid arthritis, ankylosing spondylitis, and psoriasis (6-11). A pediatric study described a particularly strong association of IBD with rheumatoid arthritis and systemic lupus in children compared with previous adult studies (6).

Several clues for the close linkage between gut and joint inflammation have been suggested. Tumor necrosis factor- $\alpha$ (TNF- $\alpha$ ) plays a key role in the pathogenesis of various arthritis diseases and IBD. Mesenchymal/myofibroblast-like cells may present the primary target of TNF- $\alpha$ in the induction of gut and joint pathology (12). Alterations in key molecules that regulate the immune response in the gut of patients with enthesitis-related JIA arthritis are similar to those with CD (13).

\footnotetext{
The first two authors contributed equally to this work.

'Department of Paediatric and Adolescent Medicine, Medical University of Vienna, Vienna, Austria; ${ }^{2}$ Department of Paediatric Gastroenterology, Great Ormond Street Hospital NHS Foundation Trust, London, UK; ${ }^{3}$ Department of Paediatrics, Gastroenterology Service, KK Women's and Children's Hospital, Singapore, Singapore; ${ }^{4}$ Translational Research Centre for Gastrointestinal Disorders, KU Leuven, Leuven, Belgium; ${ }^{5}$ Department of Histopathology, Great Ormond Street Hospital NHS Foundation Trust, London, UK; ${ }^{6}$ Department of Paediatric Rheumatology, Great Ormond Street Hospital NHS Foundation Trust, London, UK. Correspondence: Judith Pichler (judith.pichler@meduniwien.ac.at) Received 30 March 2015; accepted 2 November 2015; advance online publication 9 March 2016. doi:10.1038/pr.2016.27
} 
Changes commonly found in both groups are a high expression of E-cadherin in the gut or toll-like receptors, a T-helper type 1 (Th1) predominance in the intestinal mucosa, increased proportion of Th17 cells, and dysfunction of regulatory $\mathrm{T}$ cells (13). The alteration in function and regulation of these molecules may have an important role in initiation of these diseases. Additionally, increased intestinal permeability has been reported in patients with spondyloarthropathy and IBD, which may alter the local immune response to bacterial antigens or abnormal responses to certain microorganisms (13). There is also evidence of an increased angiogenesis. Chronic inflammation upregulates proangiogenic factors via inflammatory mediators, and angiogenesis perpetuates inflammation via continuous supply of inflammatory cells. Finally, genetic susceptibility is probably required to develop this pathology. De Vos (12) analyzed the mucosal gene expression profile in CD and spondyloarthritis and found 95 differentially expressed genes clustering in $\mathrm{CD}$ and spondyloarthritis with chronic gut inflammation.

Given the well-known association between gut and joint inflammation, surprisingly little is known about the features of gut inflammation in this group of children with severe joint disease. Mielants et al. (14) described 12 patients younger than $16 \mathrm{y}$ of age with late-onset juvenile chronic arthritis who had undergone colonoscopy with biopsies of the colonic mucosa and terminal ileum, and gut inflammation was demonstrated in nine (75\%) patients. Kokkonen et al. (15) examined the prevalence of immune activation in GI mucosa in children with JIA or connective tissue disease and found that the majority of children suffering from JIA or connective tissue disease with GI symptoms show abnormalities consistent with activation of the intestinal immune system such as UC or lymphoid nodular hyperplasia, as well in the duodenal bulb, terminal ileum, and colon with specific CD 3 lymphocytes in the intestine. Arvonen et al. $(16,17)$ could demonstrate that intestinal gamma/delta- intraepithelial lymphocytes are increased in children with JIA and suggested that lymphocyte cytotoxicity is abnormally increased in the intestinal mucosa in JIA.

The aim of this study is to evaluate GI mucosal histopathological features in a series of children with JIA who underwent endoscopic evaluation for significant GI symptoms.

\section{RESULTS}

\section{Study Cohort}

We identified 34 children ( 22 or $65 \%$ male) with a diagnosis of JIA who had undergone endoscopy for GI symptoms. One 15-y-old patient with oligoarthritis was excluded. This child had a new diagnosis of CD made at the time of endoscopy where the joint disease was the primary presentation.

The median age of onset of the remaining 33 JIA patients was $6.8 \mathrm{y}$ (range 1.5 to 15.8). The median age at time of endoscopy was of 9.2 y (range 3.3 to 16.3). The type of JIA was oligoarticular JIA in 11 (34\%) cases, polyarticular JIA in $5(15 \%)$ cases, systemic onset JIA in $8(24 \%)$ cases, and ERA in $8(24 \%)$ cases, and psoriatic JIA in $1(3 \%)$ child (Table 1$)$.
Table 1. Demographics at endoscopy

\begin{tabular}{|c|c|}
\hline Total number of children ${ }^{a}$ & $33(100 \%)$ \\
\hline Male & $21(64 \%)$ \\
\hline Median age at endoscopy (range) in years ${ }^{b}$ & $9.2(3.3-16.3)$ \\
\hline Median duration of articular symptoms in years & $6.8(1.5-15.8)$ \\
\hline HLA-27 positive/number of patients tested ${ }^{a}$ & $5 / 28(18 \%)$ \\
\hline Enthesitis-related JIA & 5 \\
\hline RF positive/number of patients tested & $4 / 28(14 \%)$ \\
\hline Polyarticular & 1 \\
\hline Enthesitis-related JIA & 1 \\
\hline Systemic & 2 \\
\hline ANA positive/number of patients tested & $7 / 28(25 \%)$ \\
\hline Oligoarticular & 3 \\
\hline Polyarticular & 2 \\
\hline Systemic & 2 \\
\hline \multicolumn{2}{|l|}{ Types of JIA } \\
\hline Oligoarticular & $11(34 \%)$ \\
\hline Polyarticular & $5(15 \%)$ \\
\hline Enthesitis-related JIA & $8(24 \%)$ \\
\hline Psoriatric & $1(3 \%)$ \\
\hline Systemic & $8(24 \%)$ \\
\hline \multicolumn{2}{|l|}{ Treatment in whole cohort } \\
\hline No treatment & $9(27 \%)$ \\
\hline Sulfasalazine & $2(6 \%)$ \\
\hline NSAID & $8(24 \%)$ \\
\hline Steroids & $7(21 \%)$ \\
\hline IMs & $15(45 \%)$ \\
\hline AZA & 2 \\
\hline MTX & 13 \\
\hline Tumor necrosis factor blocker (etanercept) & $1(3 \%)$ \\
\hline \multicolumn{2}{|l|}{ Treatment in children with intestinal inflammation } \\
\hline Steroids & $1(3 \%)$ \\
\hline NSAIDs & $4(12 \%)$ \\
\hline Sulfasalazin & $2(6 \%)$ \\
\hline Steroids and IMs & $4(12 \%)$ \\
\hline AZA & 1 \\
\hline MTX & 3 \\
\hline NSAIDs and IMs & $2(6 \%)$ \\
\hline MTX & 2 \\
\hline IMs & $7(21 \%)$ \\
\hline MTX & 7 \\
\hline Tumor necrosis factor blocker (etanercept) & $1(3 \%)$ \\
\hline
\end{tabular}


There was a correlation of age of onset with type of JIA. Children with ERA had a later age of diagnosis with median 11.2 y (range 7.6-14.2) compared to the whole cohort with median 4.6 y (range 1.5 to $15.8, P=0.002$ ).

The most common GI indications for endoscopy were persistent abdominal pain in 14 of 33 (42\%), diarrhea in 10 of 33 $(30 \%)$, faltering growth or weight loss in 6 of $33(18 \%)$, and per-rectal bleeding in 5 of $33(15 \%)$ children, and other less common GI symptoms were mouth ulcers, hematemesis, or constipation. There was no association between the clinical GI symptoms and type of JIA using univariate analyses.
In $26(79 \%)$ children, an upper and lower endoscopy was performed. In three (9\%) children, only an upper endoscopy, and in four (12\%) children, only a lower endoscopy was performed. Histological examination of gut mucosal biopsies demonstrated some gut inflammation in 28 of 33 (85\%) with 5 of 33 (15\%) having normal histology (Table 2). There were significantly more children with gut inflammation in the JIA group compared with the control group with 8 of $35(22 \%)$ children, $P<0.001$.

In the upper endoscopy, the inflammation was mostly located in the duodenum in 11 of 28 (39\%), in the stomach in

Table 2. JIA patients with abnormal gut histology, JIA types, gastrointestinal symptoms and treatment

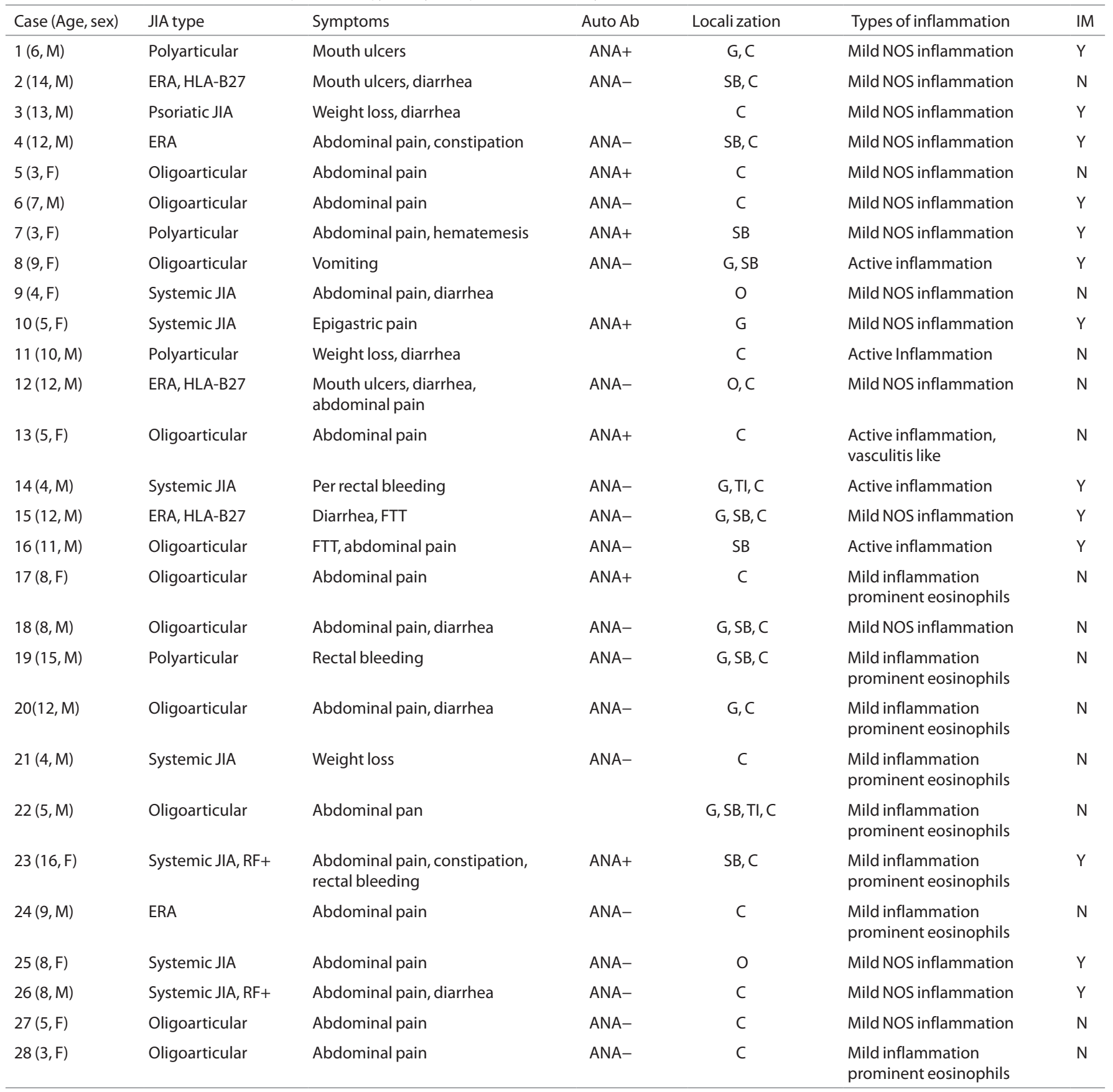

ANA, antinuclear antibody; C, colon; ERA, enthesitis-related arthritis; FTT, failure to thrive; $G$, gastric; IM, immunomodulator; IIA, juvenile idiopathic arthritis; $\mathrm{N}$, no; NOS, nonspecific; $\mathrm{O}$, esophagus; SB, small bowel; TI, terminal ileum; RF, rheumatoid factor; $Y$, yes. 


\section{Articles | Pichleretal.}

8 of $28(28 \%)$, and in both the stomach and duodenum in 3 of 28 (11\%) children.

In the lower endoscopy, inflammation was mostly located in the colon in 24 of 28 (86\%) of cases; no child had isolated inflammation in the terminal ileum. In 2 of 28 (7\%) cases, both colon and ileum were affected.

In our cohort of patients with abnormal histological features, the majority of children (11/28 (39\%)) had involvement of the colon only, 4 of 28 (14\%) had involvement of duodenum and colon, 3 of 28 (11\%) had involvement of stomach, duodenum, and colon (one of those included inflammation of the terminal ileum), 2 of 28 (7\%) had involvement of stomach and colon, and one had involvement of esophagus and colon.

Active inflammation was present in 6 of 28 (21\%) children. Fifteen (53\%) of the 28 intestinal mucosal biopsies showed nonspecific inflammation and 9 of the 28 patients (32\%) had mild inflammatory changes with predominant eosinophils (Figure 1). In five cases, this involved only the colon, in two cases involved TI and colon, and in two cases involved the duodenum and colon.

In the control group, 8 of 35 (22\%) had eosinophilic infiltrate, in all cases exclusively in the colon. There was no direct correlation between the types of JIA, gut inflammation, type of gut inflammation, clinical symptoms, and treatment using $\chi^{2}$ test.

In 28 of the 33 patients, serum autoantibody levels were available. Of the 11 of $28(39 \%)$ patients with abnormal gut histology, antinuclear antibodies were positive in 7 of $28(25 \%)$ of the patients and RF was positive in 4 of 28 (14\%) cases (two cases with systemic JIA, one case with polyarticular JIA, and one case with ERA). No gut-specific autoantibodies had been performed (e.g., anti-neutrophil cytoplasmic antibody, antiSaccharomyces cerevisiae antibody). We did not observe any direct correlation between autoantibodies positivity and types of gut inflammation using univariate analyses.

Of the 33 patients, 26 received treatment for JIA. One (3\%) child of the 29 patients with abnormal histology was on steroids

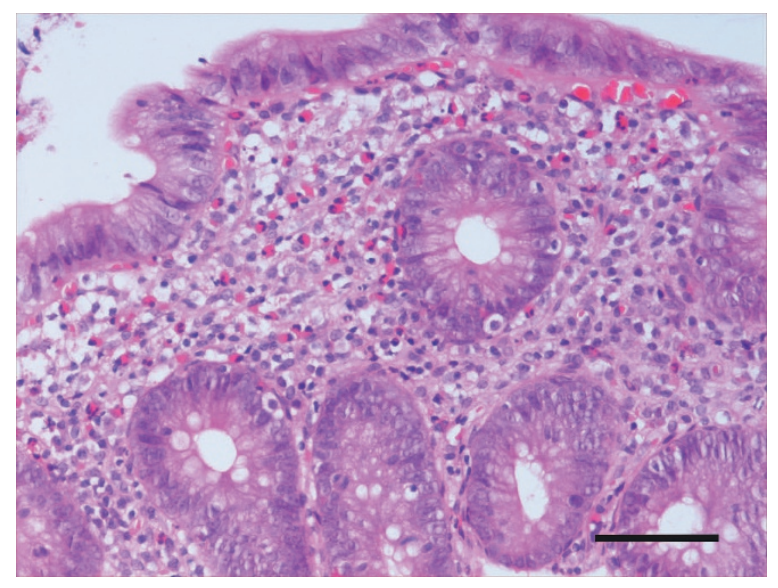

Figure 1. Photomicrograph of colonic mucosal biopsy from a child with JIA demonstrating an increase in lamina propria mild inflammatory cell density with numerous eosinophils. (Hematoxylin and eosin stain. Original magnification $\AA ̊ \sim 100$, Bar $=40 \mu \mathrm{m}$ ). only, 4 of 29 (13\%) patients were on steroids and immunomodulators namely azathioprine and methotrexate, 2 of 29 (7\%) patients were on non steroidal anti-inflammatory drugs (NSAIDs) and immunomodulators, 7 of 29 (24\%) were on immunomodulators only, and 1 of 29 (3\%) was on etanercept, a tumor necrosis factor blocker at the time of the endoscopy. In addition, there were four (13\%) children on NSAIDs and two (7\%) on sulfasalazine (Tables 1 and 2). Overall, 8 (27\%) of the 29 children with abnormal histology were not on treatment at the time of endoscopy.

Among the group of five patients with normal histology, one (20\%) child was off treatment, two (40\%) were on steroids and immunomodulators, one (20\%) on immunomodulators only, and two (40\%) were on sulfasalazine.

When immunomodulators were used, no abnormal eosinophilic infiltrate was identified compared with seven children with no treatment that had eosinophilic infiltration $(P=0.01)$. No other association of the immunosuppressive therapy with chronicity of the inflammation or the type of inflammation could be found using univariate analyses.

\section{DISCUSSION}

The findings of this study have described the histological abnormalities seen in GI mucosal biopsies in children with JIA with significant GI symptoms. The predominant pathological findings were a varying severity of mucosal inflammation ranging from mild nonspecific inflammation to active inflammation with neutrophils in $85 \%$ of the children. However, the most novel and interesting aspect of the results was the high prevalence of eosinophilia present in one third of the patients.

More than $80 \%$ of our cohort of children had abnormal mucosal gut inflammation despite almost half of them having been on immunomodulators at the time of endoscopy. The majority showed colonic inflammation, a finding similar to the previous observation of arthritis as an extraintestinal manifestation occurring more commonly in $\mathrm{CD}$ patients with large bowel evolvement $(18,19)$.

There was no correlation between gut inflammation and subtype of JIA. There were more boys than girls in this group of patients despite JIA being commoner in girls (20). This can be explained by the larger number of systemic onset JIA with $24 \%$ (where the sex incidence is equal) and also 24\% ERA patients (where boys are more often affected than girls). This could have been due to small numbers and so by chance, or GI symptoms may be commoner in these JIA subtypes.

Another speculation about overrepresentation of systemic onset or ERA could be that in most of the cases, intestinal symptoms precede or coexist with joint manifestations, but in some patients, arthritis precedes the gut manifestations, even by several years (13). This could be investigated further by prospectively collecting data on GI symptoms in a large cohort of JIA patients. Why there were a high number of ERA patients with gut involvement remains unclear.

However, Stoll et al. (21) have also reported that the median fecal calprotectin level, a specific and sensitive marker for IBD, was highest in children with ERA compared with other forms 
of JIA. Enthesitis and dactylitis in IBD patients have been studied less well than peripheral arthritis (22). The prevalence of enthesitis in patients with IBD varies from 5 to $10 \%$, it is predominantly seen in CD (23).

Prior studies have established altered microbiota and immunologic reactivity to enteric commensal organisms in IBD. Stoll et al. found a reduced abundance of Faecalibacterium in the stool of children with enthesitis-related JIA (24). Differences in the humoral responses to these bacteria may contribute to disease. So ERA patients may also have altered microbiota and immune responsiveness to enteric organisms which could influence gut inflammation (24), however, we do not have any data about microbiota to support this.

Acute inflammation occurred in 5 of 28 patients with abnormal histology. Nine out of 15 patients with mild nonspecific inflammation had been on immunomodulators that might have modified their disease activity hence presenting with a much less severe picture. Prevalence of active IBD could potentially have been even higher. Pediatric rheumatologist should be aware of IBD in any child presenting with specific subset of chronic arthritis. Evaluation of possible intestinal inflammation in children affected by chronic arthritis is crucial in order to establish correct therapeutic option, since in enteropathic arthropathies, the management of gut inflammation indirectly induces remission of articular manifestation (25). Especially a diagnosis of IBD needs to be excluded where arthritis is the most frequent extraintestinal manifestation, occurring in $7-25 \%$ of children with IBD preceding GI signs and symptoms sometimes by several years (25). The detection of an underlying IBD revealed to be crucial in order to establish the correct treatment option that led to clinical improvement (25). If histopathology might be indecisive and IBD could not be excluded, it might be reasonable in these children to perform further assessment following including imaging and mid gut assessment (26). Therefore, it might be of great importance that pediatric rheumatologists and gastroenterologists work close together. An experienced histopathologist who is aware of latest guidelines of classification and different types of IBD can additionally influence the right diagnose in an important way.

It was previously noted that in adults with ankylosing spondylitis, 5-10\% have IBD (19). Approximately 30-60\% patients with spondyloarthropathies have occult intestinal inflammation, which may be related to their ingestion of NSAIDs or associated with their rheumatic disease (18). In a prospective study of 123 patients with spondyloarthropathies who initially underwent endoscopy, intestinal evolution was evaluated by ileocolonoscopy, and an evolution to IBD was recorded in $7 \%$ of these patients (27). Despite the high frequency of gut lesions in patients with joint diseases, only a few patients are symptomatic. In a series described by Cuvelier et al. (28), only $27 \%$ of patients with histological gut inflammation had intestinal symptoms. In our cohort of JIA children who had undergone endoscopy, all have had significant GI symptoms with only a minority having normal histological findings, though there was no correlation with subtype of JIA. This study cannot comment on the prevalence of subclinical gut inflammation in JIA patients who have no GI symptoms.

Eosinophilic gut mucosal inflammation was present in nearly $30 \%$ of our JIA group and in $22 \%$ of our control group. Kokkonen et al. (15) found that intraepithelial $\mathrm{CD}^{+}, \alpha / \beta^{+}$, and $\gamma / \delta^{+}$lymphocyte counts were increased in children with JIA compared with a control group. The etiology of this reaction remains unknown, but similar features are seen in delayedtype food allergy. In line with this, Arvonen found that the number of Granzyme B-expressing intraepithelial lymphocytes was increased in duodenal mucosa in patients with JIA; hence, lymphocyte cytotoxicity is abnormally increased in the intestinal mucosa in JIA. Similar pattern of activation has been seen in food allergy and celiac disease $(16,17)$. Not only food allergy but also allergy to medications such as NSAIDs might be an explanation. However, Stoll et al. (21) found that fecal calprotectin was not associated with the use of NSAIDs in children with different types of JIA. Arvonen et al. $(16,17)$ speculated that some luminal, possibly a nutritional factor may be involved in JIA as well. In $22 \%$ of the control group with intractable constipation, there was an eosinophilic infiltrate. In what way our high eosinophilic infiltrate in the gut mucosa could play a role with the previous findings remains unclear. It could be speculated that a direct or indirect activation from intraepithelial lymphocytes might be an explanation, but further studies are needed to see whether cytotoxic activation plays any role in the pathogenesis of JIA.

A high percentage of nearly $30 \%$ eosinophilic gut mucosal inflammation was present in our JIA cohort. There were only two previous case reports describing association of eosinophilic gut disease with rheumatoid arthritis in adults $(29,30)$. The mechanism for the eosinophilic GI disorders in JIA remains to be established as well the definition of eosinophilic infiltrate in the gut mucosa. A histological finding of eosinophil infiltration (six cells-high power field (HPF)) in the lamina propria was thought to be a useful threshold for diagnosis of food protein-induced proctocolitis $(31,32)$. However, DeBrosse et al. (33) recently described that eosinophils (mean 16-20 cells-HPF) were normally observed in the GI tract of control children, especially in the colon. Functional studies of eosinophilic GI disorders are lacking and much needed to understand the physiology and pathophysiological roles of eosinophils in the GI tract (34).

Management of eosinophilic GI disorders is comprehensive and may involve routine endoscopies. Treating these children with JIA with simple dietary manipulation, in severe cases, amino acid-based elemental formulas, antihistamines, and/or thiopurines (azathioprine or 6-mercaptopurine), may be helpful in the management of their GI symptoms (35-37). In our cohort, there was a negative association with the use of immunomodulators and the presence of eosinophilic infiltrate in the intestinal mucosa. Therefore, in these patients an earlier start of treatment with immunodulators might be reasonable.

There are some limitations in this retrospective study. First, this is a highly selected group of children with JIA and GI symptoms in a tertiary centre. This does not represent the 
whole spectrum of disease in children with JIA. Secondly, there was no direct (blood test) or indirect (fecal calprotectin) evaluation of mucosal inflammation. Thirdly, we cannot provide histology results at onset of JIA and have not included previous medications. However, all our cohort were scoped when they experienced first significant GI problems, treatment which they had years before might not have influenced this inflammation.

In conclusion, our study has described gut mucosal inflammation in children with JIA who presented with GI symptoms in a tertiary centre. In addition, there was prominent eosinophilic infiltration in the intestinal mucosa of some cases. The etiology of this reaction remains unknown, with several possible explanations such as in delayed-type food allergy, allergy to medications such as NSAIDs, or a pathologic infiltration of intraepithelial lymphocytes. In what way the high eosinophilic infiltrate in the gut mucosa could play a role with the previous findings remains still unclear. Further studies are needed to see whether cytotoxic activation plays any role in the pathogenesis of JIA and to understand the physiology and pathophysiological roles of eosinophils in the GI tract.

\section{METHODS}

Patients referred to a pediatric gastroenterology centre during the period January 2002 to December 2009 who underwent GI endoscopy for GI symptoms with JIA were retrieved from hospital databases. All cases of JIA who had endoscopic examination were identified, and medical records were analyzed retrospectively. The diagnosis for JIA was made by experienced pediatric rheumatologists and classified using International League of Association for Rheumatology criteria (2).

Children with JIA with significant bowel symptoms were referred for further evaluation usually by either upper and/or lower endoscopy. Significant GI symptoms included diarrhea, constipation, vomiting, abdominal pain, weight loss, oral aphthous ulcers, or per-rectal bleeding.

Demographic data at the time of endoscopy were sex, age at start of JIA, and age when endoscopy was performed. In addition to baseline demographics, specific types of JIA, types of treatment patients were on at the time of endoscopy, presence of autoantibodies (antinuclear antibodies (ANAs) and RF) and human leukocyte antigen-B27 (HLAB27) association were reviewed.

All children who underwent endoscopy had routine mucosal biopsies taken from esophagus, stomach, duodenum, terminal ileum, and colon (cecum, ascending colon, transverse colon, descending colon, sigmoid colon, and rectum) following the departmental protocol. Routine formalin fixation, paraffin embedding, and sectioning of $4-\mu \mathrm{m}$ thick sections were performed followed by hematoxylin and eosin staining. All cases were reported as per usual clinical practice. For the purposes of this study, cases were reviewed by a single pathologist who was blinded to specific clinical information but not blinded to whether patients were included in this specific study.

Histological features as per standard histopathology reporting practice for GI mucosal biopsies (26) were recorded and compared with the clinical information. For the purposes of this article, there were additionally four categories of GI biopsy finding: (i) normal, (ii) mild nonspecific inflammation-increased lamina propria inflammatory cells density but no neutrophilic infiltration, (iii) active inflammationneutrophilic crypt or surface epithelial infiltration, (iv) prominent eosinophils. Eosinophilic esophagitis was defined by the presence of more than 15 eosinophils per HPF $(38,39)$. In the colon, although no consensus exists, we considered 30 eosinophils per HPF in the cecum and 20 eosinophils per HPF in the left colon as normal values $(39,40)$.

To compare endoscopy results, especially the incidence of eosinophilia, a control group was used. It included 35 children ( 19 or $54 \%$ male, mean age $7.9 \pm 3.6 \mathrm{y}$ (range: 2.8 to $15 \mathrm{y}$ ) with intractable constipation (defined as per European Society for Paediatric Gastroenterology, Hepatology and Nutrition guidelines (41) as chronic constipation not responding to optimum medical therapy for at least $3 \mathrm{mo}$ ) who have undergone endoscopy.

The baseline characteristics of all patients were evaluated by means of simple descriptive analysis. Continuous variables were presented as median, minimum and maximum values, and categorical data as absolute frequencies and proportions. As univariate analyses $\chi^{2}$ test or Fisher exact test was used to compare the different types of JIA or inflammation and location of inflammation. Spearman's $\rho$ test was used when appropriate. Statistical analyses were performed using SPSS (version 20.0 Mac; SPSS, Chicago, IL). All statistical tests were two-tailed using 0.05 level of significance.

The study was approved by the Clinical Audit Department of Great Ormond Street Hospital NHS Foundation Trust as a retrospective review / audit. The study was retrospectively done; therefore subject and/or parental informed consent have not been obtained.

\section{ACKNOWLEDGMENTS}

We thank Mohamed Mutalib, Marta Salach, and Alexsandra Zambrano Perez for providing us data for the control group and follow-up endoscopies.

\section{STATEMENT OF FINANCIAL SUPPORT}

Neil James Sebire is supported in part by Great Ormond Street Hospital Children's Charity (GOSHCC) and the National Institute for Health Research (NIHR) Great Ormond Street Hospital Biomedical Research Centre (GOSHBRC).

Disclosure: The authors have no conflicts of interest to disclose. There were no financial or personal relationships with other people or organizations that could have inappropriately influenced this work. There were no financial or personal relationships with any company or organization sponsoring the research at the time the research was done. There was no funding.

\section{ADDITIONAL AUTHOR INFORMATION}

J.P. carried out initial analyses, coordinated and supervised data collection, drafted the first manuscript, and approved the final manuscript. C.O. conceptualized and designed the study, carried out initial analyses, coordinated and supervised data collection, drafted the first manuscript, and approved the final manuscript. N.J.S. reviewed the histology slides and approved the final manuscript. F.K. conceptualized and designed the study and carried out initial analyses. N.S. critically reviewed the manuscript, approved the final manuscript, and gave the allergic/eosinophilic expertise. O.B. critically reviewed and revised the manuscript and approved the final manuscript. C.P. critically reviewed the manuscript, approved the final manuscript as submitted, and gave the rheumatology expertise. M.E. conceptualized and designed the study, critically reviewed and revised the manuscript, and approved the final manuscript as submitted. All authors have seen and approved the submission of this version of the manuscript and takes full responsibility for its contents.

\section{REFERENCES}

1. Weiss JE, Ilowite NT. Juvenile idiopathic arthritis. Pediatr Clin North Am 2005;52:413-42.

2. Petty RE, Southwood TR, Manners P, et al.; International League of Associations for Rheumatology. International League of Associations for Rheumatology classification of juvenile idiopathic arthritis: second revision, Edmonton, 2001. J Rheumatol 2004;31:390-2.

3. Jose FA, Garnett EA, Vittinghoff E, et al. Development of extraintestinal manifestations in pediatric patients with inflammatory bowel disease. Inflamm Bowel Dis 2009;15:63-8.

4. Diefenbach KA, Breuer CK. Pediatric inflammatory bowel disease. World J Gastroenterol 2006;12:3204-12.

5. Wordsworth P. Arthritis and inflammatory bowel disease. Curr Rheumatol Rep 2000;2:87-8.

6. Kappelman MD, Galanko JA, Porter CQ, Sandler RS. Association of paediatric inflammatory bowel disease with other immune-mediated diseases. Arch Dis Child 2011;96:1042-6.

7. Bernstein $\mathrm{CN}$, Wajda A, Blanchard JF. The clustering of other chronic inflammatory diseases in inflammatory bowel disease: a population-based study. Gastroenterology 2005;129:827-36. 
8. Cohen R, Robinson D Jr, Paramore C, Fraeman K, Renahan K, Bala M. Autoimmune disease concomitance among inflammatory bowel disease patients in the United States, 2001-2002. Inflamm Bowel Dis 2008;14:738-43.

9. Weng X, Liu L, Barcellos LF, Allison JE, Herrinton LJ. Clustering of inflammatory bowel disease with immune mediated diseases among members of a Northern California-managed care organization. Am J Gastroenterol 2007;102:1429-35.

10. Gupta G, Gelfand JM, Lewis JD. Increased risk for demyelinating diseases in patients with inflammatory bowel disease. Gastroenterology 2005;129:819-26.

11. Bardella MT, Elli L, De Matteis S, Floriani I, Torri V, Piodi L. Autoimmune disorders in patients affected by celiac sprue and inflammatory bowel disease. Ann Med 2009;41:139-43.

12. De Vos M. Joint involvement associated with inflammatory bowel disease. Dig Dis 2009;27:511-5.

13. Rodríguez-Reyna TS, Martínez-Reyes C, Yamamoto-Furusho JK. Rheumatic manifestations of inflammatory bowel disease. World J Gastroenterol 2009;15:5517-24.

14. Mielants H, Veys EM, Cuvelier C, et al. Gut inflammation in children with late onset pauciarticular juvenile chronic arthritis and evolution to adult spondyloarthropathy-a prospective study. J Rheumatol 1993;20:1567-72.

15. Kokkonen J, Arvonen M, Vähäsalo P, Karttunen TJ. Intestinal immune activation in juvenile idiopathic arthritis and connective tissue disease. Scand J Rheumatol 2007;36:386-9.

16. Arvonen $M$, Vähäsalo $P$, Turunen $S$, et al. Altered expression of intestinal human leucocyte antigen D-related and immune signalling molecules in juvenile idiopathic arthritis. Clin Exp Immunol 2012;170:266-73.

17. Arvonen M, Ikni L, Augustin M, Karttunen TJ, Vähäsalo P. Increase of duodenal and ileal mucosal cytotoxic lymphocytes in juvenile idiopathic arthritis. Clin Exp Rheumatol 2010;28:128-34.

18. Rudwaleit M, Baeten D. Ankylosing spondylitis and bowel disease. Best Pract Res Clin Rheumatol 2006;20:451-71.

19. Mielants H, Veys EM, Cuvelier C, et al. The evolution of spondyloarthropathies in relation to gut histology: III. Relation between gut and joint. J Rheumatol 1995;22:2279-84.

20. Saurenmann RK, Levin AV, Feldman BM, Laxer RM, Schneider R, Silverman ED. Risk factors for development of uveitis differ between girls and boys with juvenile idiopathic arthritis. Arthritis Rheum 2010;62:1824-8.

21. Stoll ML, Punaro M, Patel AS. Fecal calprotectin in children with the enthesitis-related arthritis subtype of juvenile idiopathic arthritis. J Rheumatol 2011;38:2274-5.

22. Brakenhoff LK, van der Heijde DM, Hommes DW, Huizinga TW, Fidder HH. The joint-gut axis in inflammatory bowel diseases. J Crohns Colitis 2010;4:257-68.

23. Brakenhoff LK, van der Heijde DM, Hommes DW. IBD and arthropathies: a practical approach to its diagnosis and management. Gut 2011;60:1426-35.

24. Stoll ML, Kumar R, Morrow CD, et al. Altered microbiota associated with abnormal humoral immune responses to commensal organisms in enthesitis-related arthritis. Arthritis Res Ther 2014;16:486.

25. Taddio A, Simonini G, Lionetti P, et al. Usefulness of wireless capsule endoscopy for detecting inflammatory bowel disease in children presenting with arthropathy. Eur J Pediatr 2011;170:1343-7.
26. Levine A, Koletzko S, Turner D, et al.; European Society of Pediatric Gastroenterology, Hepatology, and Nutrition. ESPGHAN revised porto criteria for the diagnosis of inflammatory bowel disease in children and adolescents. J Pediatr Gastroenterol Nutr 2014;58:795-806.

27. Mielants H, Veys EM, Cuvelier C, et al. The evolution of spondyloarthropathies in relation to gut histology: II. Histological aspects. J Rheumatol 1995;22:2273-8.

28. Cuvelier C, Barbatis C, Mielants H, De Vos M, Roels H, Veys E. Histopathology of intestinal inflammation related to reactive arthritis. Gut 1987;28:394-401.

29. Medina PM, Novales VG, Medina PA, et al. Eosinophilic gastroenteritis associated with rheumatoid arthritis. Its presentation as acute abdominal pain. Rev Esp Enferm Dig 1997;89:143-4.

30. Ng WF, Cohen P, Hepburn A, Hamdulay S, Carpani M, Mason JC. A case of eosinophilic enteritis and rheumatoid arthritis. Rheumatology (Oxford) 2005;44:1585-6.

31. Odze RD, Wershil BK, Leichtner AM, Antonioli DA. Allergic colitis in infants. J Pediatr 1995;126:163-70.

32. Odze RD, Bines J, Leichtner AM, Goldman H, Antonioli DA. Allergic proctocolitis in infants: a prospective clinicopathologic biopsy study. Hum Pathol 1993;24:668-74.

33. DeBrosse CW, Case JW, Putnam PE, Collins MH, Rothenberg ME. Quantity and distribution of eosinophils in the gastrointestinal tract of children. Pediatr Dev Pathol 2006;9:210-8.

34. Masterson JC, Furuta GT, Lee JJ. Update on clinical and immunological features of eosinophilic gastrointestinal diseases. Curr Opin Gastroenterol 2011;27:515-22.

35. Demetter P, Baeten D, De Keyser F, et al. Subclinical gut inflammation in spondyloarthropathy patients is associated with upregulation of the E-cadherin/catenin complex. Ann Rheum Dis 2000;59:211-6.

36. Hommel KA, Franciosi JP, Gray WN, Hente EA, Ahrens A, Rothenberg ME. Behavioral functioning and treatment adherence in pediatric eosinophilic gastrointestinal disorders. Pediatr Allergy Immunol 2012;23: 494-9.

37. Lucendo AJ. Eosinophilic diseases of the gastrointestinal tract. Scand J Gastroenterol 2010;45:1013-21.

38. Furuta GT, Liacouras CA, Collins $\mathrm{MH}$, et al.; First International Gastrointestinal Eosinophil Research Symposium (FIGERS) Subcommittees. Eosinophilic esophagitis in children and adults: a systematic review and consensus recommendations for diagnosis and treatment. Gastroenterology 2007;133:1342-63.

39. Okpara N, Aswad B, Baffy G. Eosinophilic colitis. World J Gastroenterol 2009;15:2975-9.

40. Mutalib M, Blackstock S, Evans V, et al. Eosinophilic gastrointestinal disease and inflammatory bowel disease in children: is it a disease continuum? Eur J Gastroenterol Hepatol 2015;27:20-3.

41. Tabbers MM, DiLorenzo C, Berger MY, et al.; European Society for Pediatric Gastroenterology, Hepatology, and Nutrition; North American Society for Pediatric Gastroenterology. Evaluation and treatment of functional constipation in infants and children: evidence-based recommendations from ESPGHAN and NASPGHAN. J Pediatr Gastroenterol Nutr 2014;58:258-74. 